\title{
AMENDMENTS
}

\section{Publisher Correction: Leidenfrost wheels}

Ambre Bouillant (1), Timothée Mouterde (D), Philippe Bourrianne, Antoine Lagarde, Christophe Clanet and David Quéré (D)

Correction to: Nature Physics https://doi.org/10.1038/s41567-018-0275-9, published online 10 September 2018.

In the version of this Letter originally published, the Supplementary Videos were incorrectly labelled; the descriptions of 1-4 should have gone with the videos of 6-9, and the descriptions of 5-9 should have gone with the videos of 1-5. This has now been corrected.

Published online: 1 October 2018

https://doi.org/10.1038/s41567-018-0329-z

\section{Author Correction: Polygonal motion and adaptable phototaxis via flagellar beat switching in the microswimmer Euglena gracilis}

Alan C. H. Tsang, Amy T. Lam and Ingmar H. Riedel-Kruse

Correction to: Nature Physics https://doi.org/10.1038/s41567-018-0277-7, published online 24 September 2018.

In the version of this Article originally published, the angular oscillation of amplitude in Fig. 4a was incorrectly labelled $\zeta$; it should have been $\xi$. Also, the blue line in the top-right corner of Fig. $6 \mathrm{~d}$ should not have been dash-dotted but solid. These have now been corrected in all versions of the Article.

Published online: 9 October 2018

https://doi.org/10.1038/s41567-018-0336-0

\section{Author Correction: Even-denominator fractional quantum Hall states at an isospin transition in monolayer graphene}

\author{
A. A. Zibrov, E. M. Spanton, H. Zhou, C. Kometter, T. Taniguchi, K. Watanabe (iD and A. F. Young (iD)
}

Correction to: Nature Physics https://doi.org/10.1038/s41567-018-0190-0 published online 2 July 2018.

In the version of this Article originally published, the sketch of 'PSP' in Fig. 1a was incorrect; it has now been replaced. The original and corrected versions of the figure are shown below.

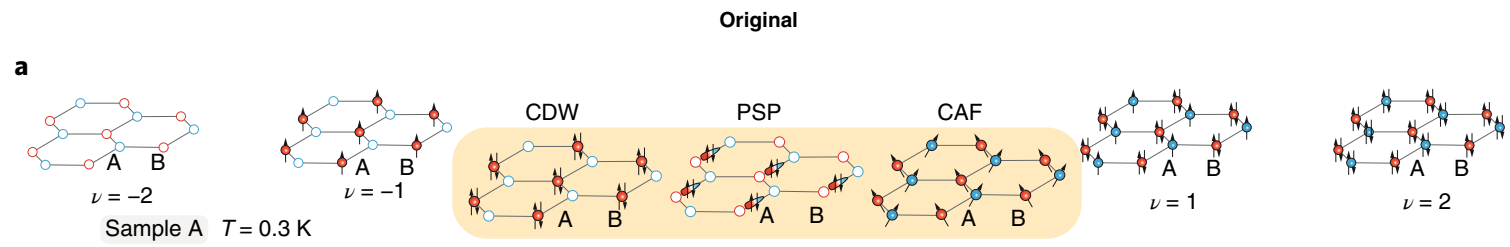

Corrected

a

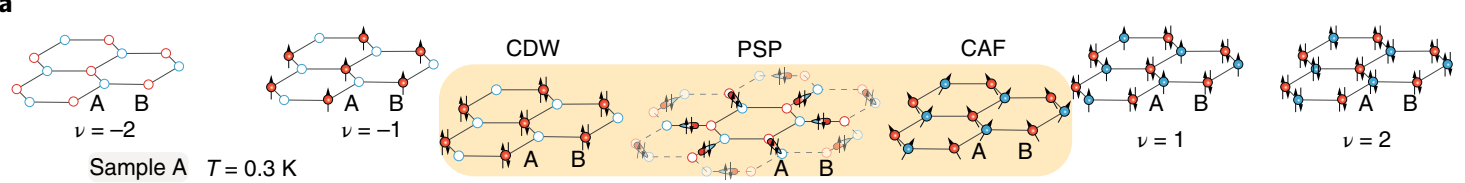

Fig. 1 | Original and Corrected. 